\title{
LORETA Neurofeedback Training
}

National Cancer Institute

\section{Source}

National Cancer Institute. LORETA Neurofeedback Training. NCI Thesaurus. Code C133083.

A type of operant conditioning that uses real-time displays of EEG to illustrate brain activity and allow a subject to self-regulate brain EEG activity. The use of low resolution electromagnetic tomography allows neurofeedback directly on deep brain areas and functions. 